\section{Diagnostic stability of acute and transient psychotic disorders in developing country settings: an overview}

\author{
Shubham Mehta \\ Department of Psychiatry, Maharaja \\ Agrasen Medical College, Agroha (Hisar), \\ Haryana, India
}

\section{Abstract}

Acute and transient psychotic disorders (ATPD), introduced in the International Classification of Diseases (ICD-10) diagnostic system in 1992, are not receiving much attention in developing countries. Therefore, the main objective of this article is to review the literature related to the diagnostic stability of ATPD in developing countries. A PubMed search was conducted to review the studies concerned with this issue in the context of developing countries, as diagnostic stability is more of a direct test of validity of psychiatric diagnoses. Four publications were found. According to the literature search, the stability percentage of the ICD-10 ATPD diagnosis is $63-100 \%$. The diagnostic shift is more commonly either towards bipolar disorder or schizophrenia, if any. Shorter duration of illness ( $<1$ month) and abrupt onset ( $<48$ hours) predict a stable diagnosis of ATPD. Based on available evidence, the diagnosis of ATPD appears to be relatively stable in developing countries. However, it is difficult to make a definitive conclusion, as there is a substantial lack of literature in developing country settings.

\section{Introduction}

Acute and transient psychotic disorder (ATPD) as a descriptive entity was recognized for the first time in 1992 in the International Classification of Diseases (ICD-10), which included it under psychotic disorders (F23) as a three digit code. ${ }^{1}$ ATPD has certain key features, such as acute onset (within 2 weeks) and rapidly changing, variable polymorphic picture, which are accepted as required criteria and stress, which is an additional criterion. Most patients experience complete recovery in 2-3 months. ${ }^{1}$ ICD-10 offers four specific and two non-specific subcategories of ATPD based on variability of clinical picture, presence of schizophrenic symptoms, and duration of the episode (Table 1).

The duration of psychotic disorders with schizophrenic symptoms is limited to 1 month because F20 schizophrenia requires a period longer than 1 month, whereas if acute psychotic disorders have polymorphic features or nonbizarre delusions, the diagnosis should be changed to F22 persistent delusional disorder after 3 months. ${ }^{1}$

Acute and transient psychotic disorder is consistently reported to occur in females between early and middle adulthood.2-4 Patients affected with ATPD do not have significant pre-morbid dysfunctions. 5 They are more likely to experience shifting polymorphic features, e.g., hallucinations or delusions of different type, which usually change in either content or intensity from day to day or within the same day. ${ }^{6}$ As a group, ATPD has different pattern of illness risk compared to schizophrenia, and different subtypes of ATPD may be genetically heterogeneous. ${ }^{7}$

The concept of ATPD has been present in psychiatry clinical practice for more than twenty years. Unfortunately, it has not received much attention from researchers, especially in developing countries, even when epidemiological studies of the incidence of acute psychosis have shown that acute and transient psychosis is ten times more common in developing countries as compared to the industrialized countries. ${ }^{8}$ The topic has been under-researched probably because of diagnostic and classification uncertainties surrounding ATPD. The present classification of the ATPD is also cumbersome and is proving to be a barrier for research and practice. Furthermore, its diagnostic stability has been questioned by various researchers.

World Health Organization is revising the ICD-10, and with ICD-11 publication expected soon, it is prudent to review the literature pertaining to the diagnostic stability of ATPD in developing countries, since this entity entails a different epidemiology and possible clinical course in such settings. ${ }^{6}$ This may enable us to understand how ATPDs affect patients chronically. This may also allow us to delineate the factors predicting the diagnostic stability or diagnostic shifts, if any.

\section{Materials and Methods}

A PubMed search was conducted using the key words acute, psychosis, and diagnostic stability and found 38 articles; while using key words acute, transient, psychosis, diagnostic stability, course, 15 articles were found. The focus was on the issue of diagnostic stability primarily because it is more of a direct test of validity of psychiatric diagnoses. The inclusion criteria for this report were publications containing data on ATPD, diagnosed as per ICD-10 criteria and assessing its diagnostic stability
Correspondence: Shubham Mehta, Department of Psychiatry, Maharaja Agrasen Medical College, Agroha (Hisar) 125047, Haryana, India.

Tel.: +91.8295.762550.

E-mail: drshubhammehta@gmail.com

Key words: Acute and transient psychosis; diagnostic stability; ICD-10.

Conflict of interest: the author declares no potential conflict of interest.

Conference presentation: the abstract was selected for poster presentation at 9th International Conference on Early Psychosis (17-19 November 2014, Tokyo, Japan).

Received for publication: 18 September 2014.

Revision received: 10 January 2015.

Accepted for publication: 3 February 2015.

This work is licensed under a Creative Commons Attribution NonCommercial 3.0 License (CC BYNC 3.0).

() Copyright S. Mehta, 2015

Licensee PAGEPress, Italy

Mental Illness 2015; 7:5640

doi:10.4081/mi.2015.5640

in developing country settings. The study had to be preferably a follow-up study as these studies include evidence of diagnostic stability and diagnostic consistency to test the validity of psychiatric diagnoses.9-11 Even so, studies based on data obtained from case records/registers of patients were also included provided they analyzed the diagnostic stability of ATPD.

\section{Results}

Following these inclusion criteria mentioned above, 4 publications ( 3 from India and one from Iran) were found suitable to be included in this report (Table 2).3,12-14

In their study, Sajith et al. ${ }^{3}$ included fortyfive patients with first episode acute polymorphic psychotic disorder without schizophrenic symptoms (APPD). Patients were followed up and assessed at regular intervals over a period of 3 years. Thirty-three cases out of 45 (73.3\%) retained their index diagnosis of APPD, while 12 cases required diagnostic revision. 10 cases changed to bipolar affective disorder and the rest to unspecified non-organic psychosis. Shorter duration of illness ( $<1$ month) and abrupt onset ( $<48$ hours) predicted a stable diagnosis of APPD.

Thangadurai et al. ${ }^{13}$ while analyzing the medical records of all patients with psychotic disorders in their set-up found that 87 patients (13.9\%) were diagnosed with acute psychosis (ICD-10 F23). Mean duration of follow-up in 
Table 1. International Classification of Diseases nomenclature of acute and transient psychotic disorders.

\begin{tabular}{llc} 
ICD-10 code & Diagnostic subcategory of AVPD (F23) & Duration, months \\
F23.0 & Acute polymorphic psychotic disorder without symptoms of schizophrenia & $<3$ \\
F23.1 & Acute polymorphic psychotic disorder with symptoms of schizophrenia & $<1$ \\
\hline F23.2 & Acute schizophrenia-like psychotic disorder & $<1$ \\
F23.3 & Other acute predominantly delusional psychotic disorders & $<3$ \\
\hline F23.8 & Other acute and transient psychotic disorders & $<3$ \\
F23.9 & Acute and transient psychotic disorders, unspecified & $<3$ \\
\hline
\end{tabular}

ICD-10, International Classification of Diseases; ATPD, acute and transient psychotic disorders.

Table 2. Studies assessing the diagnostic stability of acute and transient psychotic disorders.

\begin{tabular}{|c|c|c|c|c|}
\hline Author & Number of patients & $\begin{array}{l}\text { Duration of follow-up } \\
\text { (in months) }\end{array}$ & Study method & y percentage \\
\hline Sajith et al. ${ }^{3}$ & 45 & 36 & Prospective follow-up & 73.3 \\
\hline Amini et al. ${ }^{12}$ & 10 cases of ATPD out of total 60 cases & 12 & Prospective follow-up & 100 \\
\hline Thangadurai et al. ${ }^{13}$ & 87 & 13 & Retrospective medical record review & 64 \\
\hline Narayanaswamy et al. 14 & 57 & 24 & Retrospective medical record review & 63.2 \\
\hline
\end{tabular}

ATPD, acute and transient psychotic disorders.

their analysis was 13.2 months. The diagnosis was revised to affective disorder in 8 patients (9.2\%), schizophrenia in $23(26.4 \%)$, and 10 patients (11.5\%) presented with recurrent episodes of acute psychosis. In an another recent study from India, 14 records of 57 patients who presented with the first episode of acute and transient disorder over one year were analyzed, and the follow-up data at the end of 1 and 2 years were recorded. The records of 44 patients were available at the conclusion of one year out of which 40 patients (70.2\%) retained their diagnosis; $14 \%$ converted to bipolar disorder, $8.8 \%$ to schizophrenia and $7 \%$ were diagnosed as psychosis unspecified. Out of 43 patient records available at the end of 2 years, $63.2 \%$ retained their diagnosis; $21 \%$ were diagnosed as bipolar disorder, $8.8 \%$ as schizophrenia and $7 \%$ were diagnosed as psychosis unspecified. However, unlike the study of Sajith et al., these two studies did not specify the subcategory of ATPD. A study from Iran assessed sixty patients with first-episode psychosis at the time of discharge from hospital, and at three, six and twelve month intervals following admission. ${ }^{12}$ At each visit, two psychiatrists made consensus DSM-IV and ICD-10 diagnoses based on all the information available. Stability was recognized as consistency between diagnoses at the time of discharge and at 12 month follow-up. Forty-eight patients completed follow-up. The patients, who were diagnosed with ICD-10 acute and transient psychotic disorders initially, remained same at follow up.

\section{Discussion}

The primary purpose of this report was to assemble literature concerned with the diag- nostic stability of ATPD in developing country settings and thus to build an understanding of how this entity affects patients chronically.

Surprisingly, only four studies have evaluated the diagnostic stability of ATPD in the context of developing country settings with followup period ranging from 12-36 months. $63-100 \%$ of patients retained their diagnosis of ATPD at follow-up, suggesting a high diagnostic stability of this diagnosis. The factors which predicted a stable diagnosis of ATPD, APPD to be more specific, were shorter duration of illness ( $<1$ month) and abrupt onset $(<48 \mathrm{~h})$ as suggested by an Indian study. ${ }^{3}$

On the other hand, in industrialized nations like Europe, more than $50 \%$ of cases with ATPD tend to change diagnosis into another F2 category schizophrenia and related disorders or affective disorders as revealed in a review by Castagnini and Berrios. 15

Patients who had their diagnosis changed at follow-up most commonly received a diagnosis of either bipolar disorder or schizophrenia. These findings are similar to the studies from developed countries, which have indicated that this diagnosis changes to either schizophrenia or affective disorders. ${ }^{16,17}$

\section{Conclusions}

Based on the evidence available about the diagnostic stability of ATPD, ATPD may be more diagnostically stable in developing country settings as compared to industrialized countries. In both settings, patients with ATPD who convert to a different diagnosis at followup are either diagnosed with schizophrenia or bipolar disorder. Unfortunately, there are few studies regarding this important issue, so it is difficult to make definitive conclusions. Also, the studies reviewed in this report have differences in design (prospective $v s$. retrospective study designs) and length of follow-ups. Therefore, meaningful comparison is difficult. Future research is required to further elucidate this diagnostic dilemma because certain important questions still remain unanswered: i) What are the biological validators of the diagnosis of ATPD, considering the diagnostic stability of ATPD to be as high as $63-100 \%$ ?ii) What are the standard guidelines for the treatment of ATPD? Antipsychotics are currently used but there is no evidence base for this. iii) Considering high mortality in ATPD particularly from suicide, 18 what prevention strategies can be framed to reduce this?

These questions can serve as guide for the future research.

\section{References}

1. World Health Organization. The ICD-10 classification of mental and behavioral disorders. Geneva: World Health Organization; 1992.

2. Susser E, Wanderling J. Epidemiology of non-affective acute remitting psychosis: sex and socio-cultural setting. Arch Gen Psychiatry 1994; 51:294-301.

3. Sajith SG, Chandrasekaran R, Sadanandan Unni KE, Sahai A. Acute polymorphic psychotic disorder: diagnostic stability over 3 years. Acta Psychiatr Scand 2002;105:1049.

4. Singh SP, Burns T, Amin S, et al. Acute and transient psychotic disorders: precursors, epidemiology, course and outcome. Br J Psychiatry 2004;185:452-9.

5. Mehta S, Tyagi A, Swami MK, et al. Onset 
of acute and transient psychotic disorder in India: a study of sociodemographics and factors affecting its outcome. East Asian Arch Psychiatry 2014; 24:75-80.

6. Farooq S. Is acute and transient psychotic disorder (ATPD) mini schizophrenia? The evidence from phenomenology and epidemiology. Psychiatr Danub 2012;24:311-5.

7. Das SK, Malhotra S, Basu D. Family study of acute and transient psychotic disorders: comparison with schizophrenia. Soc Psychiatry Psychiatr Epidemiol 1999;34: 328-32.

8. Indian Council of Medical Research (ICMR). Final report of phenomenology and natural history of acute psychosis. New Delhi: ICMR; 1985.

9. Robins E, Guze S. Establishment of diagnostic validity in psychiatric illness: its application to schizophrenia. Am J Psychiatry 1970;126:983-7.
10. Kendler KS. The nosologic validity of paranoia (simple delusional disorder). Arch Gen Psychiatry 1980;37:699-706.

11. Andreasen, NC. The validation of psychiatric diagnosis: new models and approaches (editorial). Am J Psychiatry 1995;152: 161-2.

12. Amini H, Alaghband-rad J, Omid A, et al. Diagnostic stability in patients with first episode psychosis. Australas Psychiatry 2005;13:388-92.

13. Thangadurai $P$, Gopalakrishnan R, Kurian S, Jacob KS. Diagnostic stability and status of acute and transient psychotic disorders. Br J Psychiatry 2006;188:293.

14. Narayanaswamy JC, Shanmugam VH, Raveendranathan D, et al. Short-term diagnostic stability of acute psychosis: data from a tertiary care psychiatric center in South India. Indian J Psychol Med 2012; 34:176-8.
15. Castagnini A, Berrios GE. Acute and transient psychotic disorders (ICD-10 F23): a review from a European perspective. Eur Arch Psychiatry Clin Neurosci 2009;259: 433-43.

16. Aadamsoo K, Saluveer E, Kuunarpuu H, et al. Diagnostic stability over 2 years in patients with acute and transient psychotic disorders. Nord J Psychiatry 2011;65: 381-8.

17. Marneros A, Pillmann F, Haring A, et al. The relation of acute and transient psychotic disorder (ICD-10 F23) to bipolar schizoaffective disorder. J Psychiatr Res 2002;36:165-71.

18. Castagnini AC, Bertelsen A. Mortality and causes of death of acute and transient psychotic disorders. Soc Psychiatry Psychiatr Epidemiol 2011;46:1013-7. 